\title{
Raman scattering in cluster-deposited nanogranular silicon films
}

\author{
M. J. Konstantinović, S. Bersier, X. Wang, M. Hayne, P. Lievens, R. E. Silverans, and V. V. Moshchalkov \\ Laboratorium voor Vaste-Stoffysica en Magnetism, Katholieke Universiteit Leuven, Celestijnenlaan 200 D, Leuven 3001, Belgium
}

(Received 18 June 2002; published 16 October 2002)

\begin{abstract}
We study nanograin size confinement effects, and the effect of the increase of local temperature on the first-order Raman spectrum in silicon nanogranular films obtained by cluster deposition. The local temperature was monitored by measuring the Stokes/antiStokes peak ratio with the laser power up to $\sim 20 \mathrm{~kW} / \mathrm{cm}^{2}$. We find large energy shifts, up to $30 \mathrm{~cm}^{-1}$, and broadenings, up to $20 \mathrm{~cm}^{-1}$, of the Raman-active mode, which we attribute to both laser heating and confinement effects. The phonon softening and phonon linewidth are calculated using a phenomenological model which takes into account disorder effects through the breakdown of the $k=0$ Raman-scattering selection rule, and also anharmonicity, which is incorporated through the threeand four- phonon decay processes. Very good agreement with experimental data is obtained for calculated spectra with nanograin sizes of about $10 \mathrm{~nm}$, and with an increase in the anisotropy constants with respect to those of bulk silicon.
\end{abstract}

DOI: 10.1103/PhysRevB.66.161311

PACS number(s): 78.30.Am, 78.20.-e, 78.66.Db

The expectation that reducing the dimensions of silicon (Si) nanocrystals can possibly turn this material from an indirect into a direct band-gap system triggered much research in the field of $\mathrm{Si}$ optoelectronics. The discovery ${ }^{1}$ of strong photoluminescence (PL) in Si nanocrystals with sizes of about 2-5 $\mathrm{nm}$ indicated the importance of quantum size effects, and opened the possibility for new technological applications. It has been reported ${ }^{2,3}$ that confinement effects in silicon nanostructures cause the increase of the silicon band gap, and shift the PL into the visible energy range. However, the small crystal size also limits experimental investigations. One of the main challenges is to determine and control the nanocrystal size distribution, which is important for the formulation of a quantum mechanical description of the system. Raman scattering can be used to measure the shift of the Raman lines in nanocrystals with respect to those in the bulk, even though the laser spot exceeds particle sizes by several orders of magnitude.

In general, the increase of disorder, produced by the small particle volume, results in breaking the wave-vector Ramanscattering selection rule $(\mathbf{k}=0)$ and causes the softening and asymmetric broadening of the phonon modes in the Raman spectra. ${ }^{4,5}$ In various experiments of this kind ${ }^{6-13}$ the average size of $\mathrm{Si}$ nanocrystals is determined from the shift and broadening of the $T_{2 g}$ optically active phonon mode. Even though the large phonon softening has been demonstrated experimentally, and was assumed to be a result of the sizerelated increase of disorder, these studies showed poor agreement between theory and experiment concerning the phonon line shapes. Our investigation is partially motivated by such an apparent disagreement. A related problem is the heat dissipation, which in very small systems can be quite different from that in macroscopically large volumes. This raises questions about the role played by heating effects and the actual local temperature of the nanocrystals during the Ramanscattering measurements.

Here, we report measurements of the temperature dependence of the first-order Raman-active phonon signal in $\mathrm{Si}$ cluster-deposited nanograin films induced by varying the laser light power. First, we show that a moderately small change of laser power induces a large increase in the cluster temperature, and we argue that this effect becomes more pronounced in smaller grains and/or thinner cluster films. Second, from the comparison between measured and calculated Raman spectra we show that the frequency shift and the broadening are the consequence of the subtle interplay between local heating effects and confinement effects due to the nanocrystalline size.

The Si films were obtained by low-energy cluster beam deposition ${ }^{14,15}$ of nanometer-sized gas phase silicon clusters produced with a laser vaporization source. ${ }^{16}$ Size distributions measured by time-of-flight mass spectrometry show that clusters are composed from a few up to $1000 \mathrm{Si}$ atoms, with a maximum production for $\sim 600$ atoms. The clusters are deposited, without any mass selection, on a mica substrate with an impact energy of about $0.5 \mathrm{eV} /$ atom, resulting in coagulated and aggregated nanogranular films with a low density, i.e., they are highly porous. ${ }^{17}$ Scanning electron microscopy, Fig. 1(a), and optical microscopy both show bright grains of material with a range of sizes $<5 \mu \mathrm{m}$ on top of a dark film. However, ambient atomic force microscopy (AFM) measurements in the air [Fig. 1(b)] reveal a continuous disordered film with feature sizes down to the microscope resolution of $50 \mathrm{~nm}$. For the remainder of this paper we will refer to the micron-sized granular structure as "grain," and to the nanogranular building blocks with a typical size of few tens of nanometer as "nanograin." The latter results from a partial coagulation of the gas phase clusters upon deposition. . $^{4,15,17}$

Raman spectra were measured in the backscattering configuration using a micro-Raman system with a DILOR triple monochromator including a liquid-nitrogen cooled chargecoupled device detector. The 514.5-nm argon-ion laser line was used as an excitation source. Laser powers at the sample surface were varied from 20 to $700 \mu \mathrm{W}$, with a spot size of about $2 \mu \mathrm{m}$.

Micro-Raman measurements were taken from isolated grains (bright spots) with various sizes, and from the dark areas of the film. Typical Raman spectra are presented in Fig. 2. For spectra taken at bright spots we observe a sharp first- 
a)
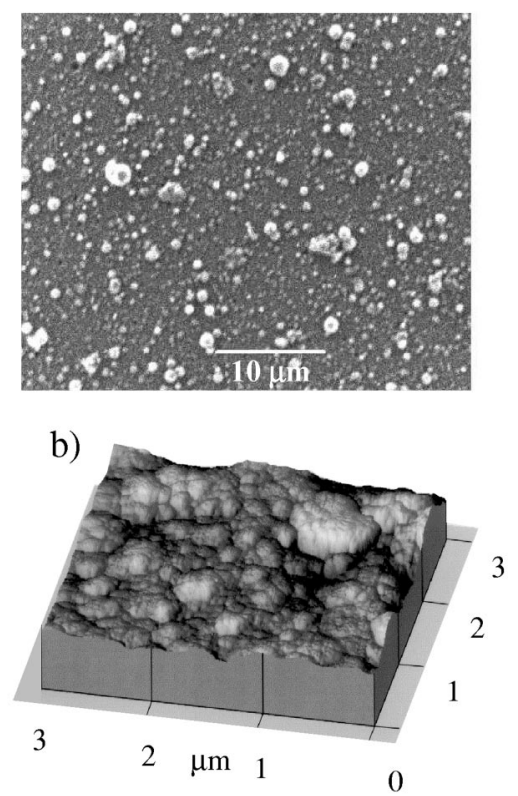

FIG. 1. (a) Silicon clusters deposited on mica as observed by (a) scanning electron microscopy, showing a micron-sized grain structure, and (b) atomic force microscopy, showing a tens-ofnanometer-sized nanograin structure.

order crystalline Si peak, while for spectra taken at dark spots the appearance of broad features indicates a fully disordered (amorphous) film structure.

The Stokes and anti-Stokes Raman spectra of Si films, measured with various laser powers are shown in Fig. 3. The results are fully reproducible whenever the laser power is increased or decreased, and repeated over many weeks. With increasing laser power, the $521-\mathrm{cm}^{-1}$ phonon mode decreases in energy and broadens. The softening and broaden-

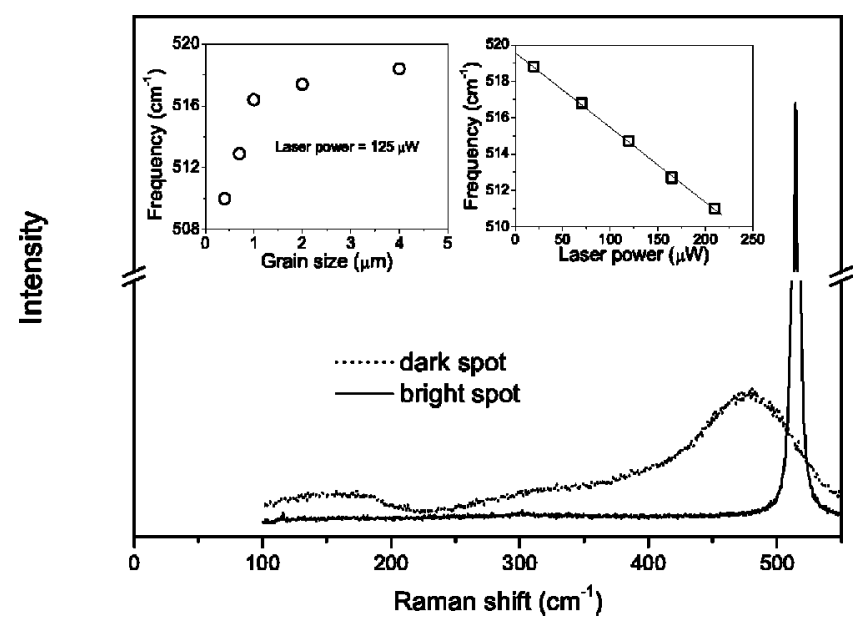

FIG. 2. Room-temperature Raman spectra taken at bright spots (full line) and in the dark area (dotted line). Left inset: Phonon frequency of the Raman spectra versus grain size. Right inset: Phonon frequency versus laser power for a grain size of about $0.4 \mu \mathrm{m}$. The squares are experimental data, and the full line is a linear fit extrapolated to zero power.

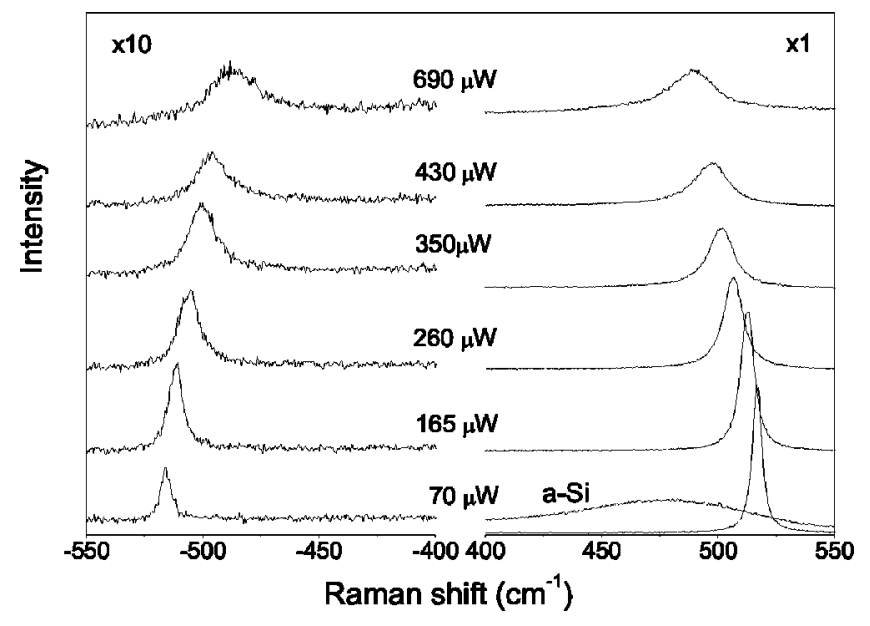

FIG. 3. Stokes and anti-Stokes Raman spectra for various laser powers and a grain size of about $0.4 \mu \mathrm{m}$. The signal from a black region attributed to amorphous $\mathrm{Si}(a-\mathrm{Si})$ is also shown for comparison.

ing are large, up to $31 \mathrm{~cm}^{-1}$ and $22 \mathrm{~cm}^{-1}$, respectively, for the highest laser power used of about $690 \mu \mathrm{W}$ (Fig. 3). It can be also seen from Fig. 3 that the intensity ratio between the Stokes and anti-Stokes part of the spectrum decreases as the laser power increases. This implies a dramatic change in the local temperature of the grains, as might be expected in micro-Raman experiments, where the laser light is focused on the micrometer-size area. Furthermore, the heating rate and the equilibrium temperature of the grains are very sensitive to the heat transfer regime, which depends upon the grain size, and the spectral acquisition times. To illustrate this we plot the phonon frequency versus grain size for a fixed laser power in the left-hand inset of Fig. 2. The frequency shift increases dramatically in smaller grains, consistent with a large increase of the heating rate. These data therefore imply that the shift of the Raman line may be obtained simply by heating rather than by a decrease in nanocrystalline size. This is most clearly demonstrated in the right-hand inset of Fig. 2, which reveals a linear relationship between the size of the Raman shift and incident laser power. The grain sizes are quite large, thus raising the question of how this effect may further increase as the grain size is reduced, and how the actual temperature of the Si grains may be controlled in such a regime.

Another important effect, directly related to the increase of the local temperature, concerns the phonon line shapes. Our Raman spectra show that the phonon line shapes are more or less symmetric regardless of the frequency shifts and the broadening (Fig. 3). To be more precise, we find a slight asymmetry, which is also present in previously reported spectra, and is much smaller than that expected from the disorder scattering model used to determine nanocrystal sizes. ${ }^{21}$ Thus, in the following part of the paper we describe the Raman spectra which we have calculated including anharmonic effects (in order to account for the temperature change) in the disorder scattering model, and show that symmetrical line shapes of the first-order silicon Raman spectra indicate the presence of anharmonicity and confirm the im- 


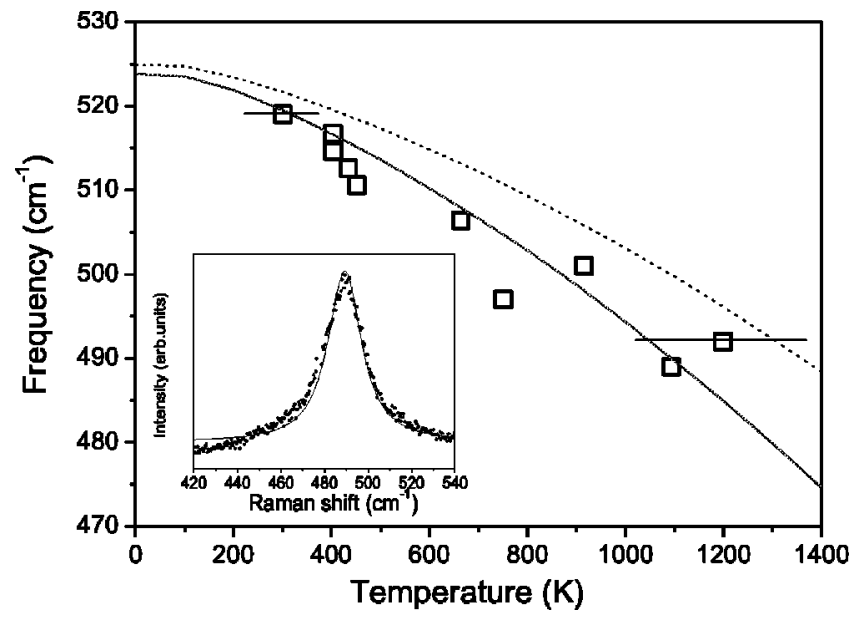

FIG. 4. Si-phonon frequency versus temperature in our experiment (squares), bulk silicon (dotted line). The calculated phonon frequency shifts, assuming a 10-nm Si nanograin with included anharmonicity, is shown with a full line, see text. The inset shows measured and calculated Raman spectra for a grain size of about $0.4 \mu \mathrm{m}$ and a laser power of $690 \mu \mathrm{W}$.

portance of the local heating effects in our cluster-deposited films.

A convenient way to determine the local temperature of the silicon grains is to use the Stokes/anti-Stokes intensity ratio which depends on temperature through the BoseEinstein occupation number: $I_{\text {Stokes }} / I_{\text {anti-Sokes }}$ $=$ const $\times e^{\hbar \omega / k_{B} T}$. We should note that this formula for const $\sim 1$ is valid for equilibrium conditions and in the adiabatic limit, which are not fulfilled in our case. Because of this, the determined temperatures are always underestimated. A similar effect is also observed for the bulk silicon, ${ }^{18}$ and we used the value const 3 to normalize the lowesttemperature experimental point to $300 \mathrm{~K}$. Due to that and also due to the lack of an additional temperature check based on an alternative method, the error bars are quite large in the case of the laser heating. Nevertheless, this uncertainty does not influence our main conclusion that the phonon softening, together with symmetrical line shapes, is almost entirely the result of the local-heating effects.

In Fig. 4 we compare the temperature dependence of the phonon frequency shifts in silicon nanograins with those in the bulk. The open squares are the phonon frequencies obtained from the Raman spectra already presented in Fig. 3. The comparison reveals two important features: (i) All points lie below the curve obtained for bulk silicon (dotted line). ${ }^{18}$ (ii) The difference between the cluster-deposited film (solid line) and the bulk curves increases at higher temperatures. We shall show that the first observation is related to the nanograin size effect, while the second results from the increase of anharmonicity in nanocrystalline $\mathrm{Si}$ with respect to bulk silicon. Here, one may argue that even for the lowest laser light power we do not know the actual temperature, which is certainly true. However, we can estimate the value of the phonon frequency at zero laser power, by extrapolating the measured frequency changes versus laser power down to zero. This is shown in the right-hand inset of Fig. 2. The extrapolated value is found to be smaller than the pho- non frequency for bulk silicon at $300 \mathrm{~K}$, by about $1.5 \mathrm{~cm}^{-1}$, which is clearly a size-related effect. Without anharmonicity, this shift correspond to a nanocrystalline Si size of about 10 nm. ${ }^{21}$ We now calculate the Raman spectra using the standard size-disorder model, 4,21

$$
I(\omega)=\int_{0}^{1} \frac{e^{-k^{2} L^{2} / 4 a^{2}}}{[\omega-\omega(k, T)]^{2}+[\Gamma(T) / 2]^{2}} d^{3} k .
$$

Anharmonicity is incorporated through the three- and four-phonon decay channels in the scattering process, ${ }^{18}$

$$
\begin{gathered}
\omega(k, T)=\omega(k)+\Delta(T) \\
\Delta(T)=C\left[1+\frac{2}{e^{\hbar \omega / 2 k_{B} T}-1}\right]+D\left[1+\frac{3}{e^{\hbar \omega / 3 k_{B} T}-1}\right. \\
\left.+\frac{3}{\left(e^{\hbar \omega / 3 k_{B} T}-1\right)^{2}}\right], \\
\Gamma(T)=A\left[1+\frac{2}{e^{\hbar \omega / 2 k_{B} T}-1}\right]+B\left[1+\frac{3}{e^{\hbar \omega / 3 k_{B} T}-1}\right. \\
\left.+\frac{3}{\left(e^{\hbar \omega / 3 k_{B} T}-1\right)^{2}}\right] .
\end{gathered}
$$

Here, $L$ corresponds to the Si nanograin size, $a$ is the lattice constant, $\omega(k)=\sqrt{[1.7+\cos (\pi k / 2)] 10^{5}} \mathrm{~cm}^{-1}$ is the phonon dispersion in silicon at $T=300 \mathrm{~K}, \Gamma(T)$ is the mode line width, and $A, B, C, D$ are anharmonic constants. This is a simplified model which considers only the decay process into two phonons of equal frequency (the inclusion of other decay processes does not change our conclusions qualitatively), and more rigorous treatments exist. ${ }^{19,20}$ A comparison between calculated and measured Raman spectra is shown in the inset of Fig. 4 for the spectra with the largest frequency shift, and with the largest broadening. The calculated curve is obtained by taking $L=10 \mathrm{~nm}$ for the nanograin size, and with the anharmonic constants $A=1.683, B=0.136, C$ $=-3.996$, and $D=-0.235 \mathrm{~cm}^{-1}$. The agreement between calculation and experiment is very good at higher frequencies. We believe that the slight disagreement at lower frequencies is caused by the contribution of the Raman intensity from the amorphous phase, which is observed as a pronounced broad structure centered around $480 \mathrm{~cm}^{-1}$, see Fig. 2 . For the elevated temperatures the crystalline peak decreases in intensity, and so becomes comparable with the amorphous signal coming from the underlying part of the film.

It is interesting to note that similar asymmetric Raman spectra are already reported in Ref. 9. There, the authors assigned the frequency shift (together with a small asymmetry) as the evidence of the (2-5)-nm cluster formation in the silicon film. However, in their case the calculated spectra show a strong disagreement with the experiment on the highfrequency side of the peak where the spectral cutoff, due to size-related density of state effect, should be observed. 
Therefore, it is likely that the Raman spectra in Ref. 9 in fact reflect just the local temperature rising. The full line in Fig. 4 represents the temperature dependence of the phonon frequency in 10-nm silicon nanograins. It shows reasonably good agreement with the experimental data. The deviation from the bulk curve can be explained by assuming the increase of the anharmonic constants of about $30 \%$. In addition, as is evident from the inset of Fig. 4, the calculated broadenings are also in a very good agreement with measurements. More importantly, the increase of the anharmonic constants ( $C$ and $D$ ) used in our calculation of the broadenings $\Gamma(T)$ (with respect to bulk $\mathrm{Si}$ ) is the same as the increase of the anharmonic constants $(A$ and $B)$ used to determine the frequency shifts. This gives a strong additional argument for the validity of our interpretation.

To summarize, we have studied the effect of confinement and temperature on the first-order Raman-active phonon in silicon nanograin films. An analysis is presented using a sizerelated disorder model with the inclusion of anharmonicity. We find that the frequency shift and the broadening of the $T_{2 g}$ mode in the Raman spectra are the consequence of both size-related and local-heating effects. Very good agreement with experimental data is obtained for calculated spectra with nanograin sizes of about $10 \mathrm{~nm}$, and with an increase in the anisotropy constants in nanograins with respect to those of bulk silicon.

This work was supported by the FWO-Vlaanderen, the Flemish GOA, and the Belgian IUAP programs. We are thankful to A. Volodin for the AFM images. M.J.K. thanks the DWTC. S.B. thanks the Swiss National Science Foundation.
${ }^{1}$ L.T. Canham, Appl. Phys. Lett. 57, 1046 (1990).

${ }^{2}$ L.T. Canham, Phys. Status Solidi B 190, 9 (1995).

${ }^{3}$ M.V. Wolkin, J. Jorne, P.M. Fauchet, G. Allan, and C. Delerue, Phys. Rev. Lett. 82, 197 (1999).

${ }^{4}$ R. Shuker and R.W. Gammon, Phys. Rev. Lett. 25, 222 (1970).

${ }^{5}$ H. Richter, Z.P. Wang, and L. Ley, Solid State Commun. 39, 625 (1981).

${ }^{6}$ Z. Iqbal, A.R. Webb, and S. Vepřek, Appl. Phys. Lett. 36, 163 (1980).

${ }^{7}$ Z. Sui, P.P. Leong, I.P. Herman, G.S. Higashi, and H. Temkin, Appl. Phys. Lett. 60, 2086 (1992).

${ }^{8}$ R. Tsu, H. Shen, and M. Dutta, Appl. Phys. Lett. 60, 6 (1992).

${ }^{9}$ Y. Kanemitsu, H. Uto, Y. Masumoto, T. Matsumoto, T. Futagi, and H. Mimura, Phys. Rev. B 48, 2827 (1993).

${ }^{10}$ Ch. Osadnik, S. Vepřek, and I. Gregora, Thin Solid Films 337, 148 (1999).

${ }^{11}$ P. Mishra, and K.P. Jain, Phys. Rev. B 62, 14790 (2000).

${ }^{12}$ K.K. Tiong, P.M. Amirtharaj, F.H. Pollak, and D.E. Aspnes, Appl. Phys. Lett. 44, 122 (1984).
${ }^{13}$ M. Holtz, R. Zallen, O. Brafman, and S. Matteson, Phys. Rev. B 37, 4609 (1988).

${ }^{14}$ A. Perez, P. Mélion, V. Dupuis, P. Jensen, B. Prével, J. Tuaillon, L. Bardotti, C. Martet, M. Trelleux, M. Broyer, M. Pellarin, J.L. Vaille, B. Palpant, and J. Lermé, J. Phys. D 30, 709 (1997).

${ }^{15}$ P. Jensen, Rev. Mod. Phys. 71, 1695 (1999).

${ }^{16}$ W. Bouwen, P. Thoen, F. Vanhoutte, S. Bouckaert, F. Despa, H. Weidele, and R.E. Silverans, Rev. Sci. Instrum. 71, 54 (1999).

${ }^{17}$ W. Bouwen, E. Kunnen, K. Temst, P. Thoen, M.J. Van Bael, F. Vanhoutte, H. Weidele, P. Lievens, and R.E. Silverans, Thin Solid Films 354, 87 (1999).

${ }^{18}$ M. Balkanski, R.F. Wallis, and E. Haro, Phys. Rev. B 28, 1928 (1983).

${ }^{19}$ J. Menéndez and M. Cardona, Phys. Rev. B 29, 2051 (1984).

${ }^{20}$ A. Debernardi, S. Baroni, and E. Molinari, Phys. Rev. Lett. 75, 1819 (1995).

${ }^{21}$ I.H. Campbell and P.M. Fauchet, Solid State Commun. 58, 739 (1984). 